\title{
List of tables
}

Tab. 1: Hengeveld's lexeme classes and their respective functions (Hengeveld 2013: 32) - 14

Tab. 2: Croft's conceptual space for the parts of speech (Croft 2000: 89) - 16

Tab. 3: Chinese word classification systems since Ma (1898) (cf. Bai 2011: 216) - 37

Tab. 4: Examples of occurrence frequencies of nouns in both $\mathrm{N}$ - and $\mathrm{V}$-functions in thirteen classical texts (Zhang 2005: 182; pīnyīn glosses added) -49

Tab. 5: Most common intransitive and transitive argument structure constructions in Classical Chinese - 82

Tab. 6: Morphological differentiation between intransitive and causative (transitive) verbs through ${ }^{*} s$ - in Old Chinese (examples from Mei 2012: 12; cf. Sagart and Baxter 2012) - 85

Tab. 7: Morphological differentiation between parts of speech through *s- in Old Chinese and Middle Chinese (examples from Sagart and Baxter 2012: 41 and Sagart 1999: 63) -85

Tab. 8: Semantic type shifts of action-denoting lexemes in nominal function -126

Tab. 9: Semantic type shifts of object-denoting lexemes in verbal function -127

Tab. 10: Metaphorical mapping from JOURNEY to LOVE (Kövecses 2010: 9) - 193

Tab. 11: Distributions of the word form 齒 chĭ- 237

Tab. 12: Distributions of the lexeme 背 bèi - 242

Tab. 13: Distributions of the lexeme 祖 $z \check{u}-244$ 
